\title{
On the puzzling feature of the silence of precursory electromagnetic emissions
}

\author{
K. Eftaxias ${ }^{1}$, S. M. Potirakis ${ }^{2}$, and T. Chelidze ${ }^{3}$ \\ ${ }^{1}$ Department of Physics, Section of Solid State Physics, University of Athens, Panepistimiopolis, 15784 Zografos, \\ Athens, Greece \\ ${ }^{2}$ Department of Electronics Engineering, Technological Education Institute (TEI) of Piraeus, 250 Thivon \& P. Ralli, \\ 12244 Aigaleo, Athens, Greece \\ ${ }^{3}$ M. Nodia Institute of Geophysics at the I. Javakhishvili Tbilisi State University, 1 Alexidze str., Tbilisi 0171, Georgia \\ Correspondence to: K. Eftaxias (ceftax @ phys.uoa.gr)
}

Received: 3 December 2012 - Published in Nat. Hazards Earth Syst. Sci. Discuss.: Revised: 5 July 2013 - Accepted: 1 August 2013 - Published: 27 September 2013

\begin{abstract}
It has been suggested that fracture-induced $\mathrm{MHz}-$ $\mathrm{kHz}$ electromagnetic emissions (EME), which emerge from a few days up to a few hours before the main seismic shock occurrence permit a real-time monitoring of the damage process during the last stages of earthquake preparation, as it happens at the laboratory scale. Despite fairly abundant evidence, electromagnetic (EM) precursors have not been adequately accepted as credible physical phenomena. These negative views are enhanced by the fact that certain "puzzling features" are repetitively observed in candidate fractureinduced pre-seismic EME. More precisely, EM silence in all frequency bands appears before the main seismic shock occurrence, as well as during the aftershock period. Actually, the view that "acceptance of "precursive" EM signals without convincing co-seismic signals should not be expected" seems to be reasonable. In this work we focus on this point. We examine whether the aforementioned features of EM silence are really puzzling ones or, instead, reflect well-documented characteristic features of the fracture process, in terms of universal structural patterns of the fracture process, recent laboratory experiments, numerical and theoretical studies of fracture dynamics, critical phenomena, percolation theory, and micromechanics of granular materials. Our analysis shows that these features should not be considered puzzling.
\end{abstract}

\section{Introduction}

In recent years, the wind prevailing in the scientific community does not appear to be favorable for earthquake (EQ) prediction research, in particular for the research of short-term prediction (Uyeda et al., 2009). Sometimes the arguments have been extended to the extreme claim that any precursory activity is impossible (Geller et al., 1997). Considering the difficulties associated with such factors as the highly complex nature and rarity of large EQs and subtleties of possible pre-seismic signatures, the present negative views are not groundless.

An EQ is a sudden mechanical failure in the Earth's crust, which has heterogeneous structures. The employment of basic principles of fracture mechanics is a challenging field for understanding the EQ preparation process. A vital problem in material science is the identification of precursors of macroscopic defects. Fracture-induced physical fields allow for a real-time monitoring of damage evolution in materials during mechanical loading. More specifically, electromagnetic emissions (EME) and acoustic emissions (AE) in a wide frequency spectrum ranging from the $\mathrm{kHz}$ to the $\mathrm{MHz}$ bands are produced by opening cracks, which can be considered as the so-called precursors of general fracture. Recently, improvements in the MHz-kHz EME technique have permitted a real-time monitoring of the fracture process (Fukui et al., 2005; Kumar and Misra, 2007; Chauhan and Ashok Misra, 2008; Baddari et al., 1999, 2011; Baddari and Frolov, 2010; Lacidogna et al., 2010; Schiavi et al., 2011; Carpinteri et al., 2012). However, the MHz-kHz electromagnetic (EM) precursors are detectable not only at the laboratory but also at the geological scale; a stressed rock behaves like a stress-EM transducer (Sadovski, 1982; Hayakawa and Fujinawa, 1994; Gokhberg et al., 1995; Hayakawa, 1999, 2009; Hayakawa and Molchanov, 2002; Eftaxias et al., 2007, 2011; Eftaxias, 
2012; Molchanov and Hayakawa, 2008). The idea that the fracture-induced $\mathrm{MHz}-\mathrm{kHz}$ EM fields should also permit the monitoring of the gradual damage of stressed materials in the Earth's crust, as happens in the laboratory experiments, in real time and step by step, seems to be justified: the aspect of self-affine nature of faulting and fracture is well documented. An interesting experimental research program would be the parallel monitoring of the corresponding observable manifestations of both laboratory- and geophysical-scale fracture phenomena. Based on this idea we have installed a field experimental network using the same instrumentation as in laboratory experiments for the recording of geophysical-scale EME. An exemplary telemetric station, as part of this network, has been operating on Zante Island (Greece) since 1994, mainly aiming at the detection of $\mathrm{kHz}-\mathrm{MHz}$ EM precursors. It has been installed in a carefully selected mountainous site in the southwest part of the island $\left(37.76^{\circ} \mathrm{N}\right.$, $20.76^{\circ} \mathrm{E}$ ), providing low EM background noise. The complete measurement system is comprised of (i) six loop antennas detecting the three components (EW, NS, and vertical) of the variations of the magnetic field at 3 and $10 \mathrm{kHz}$, respectively; (ii) three vertical $\lambda / 2$ electric dipole antennas detecting the electric field variations at 41,54 , and $135 \mathrm{MHz}$, respectively; and (iii) other magnetic and electromagnetic sensors. All the time series are sampled once per second, i.e., with a sampling frequency of $1 \mathrm{~Hz}$.

"Are there credible EM EQ precursors?" This is a question debated in the science community (Eftaxias, 2012). Despite fairly abundant evidence, EM precursors have not been adequately accepted as real physical quantities (Morgounov and Shakhraman'yan, 1996; Uyeda et al., 2009; Hayakawa and Hobara, 2010). There may be legitimate reasons for the critical views concerning the reliability of EM precursors: the degree to which we can predict a phenomenon is often measured by how well we understand it. However, many questions about the fracture processes remain standing. Specifically, many aspects of EQ generation remain yet to be understood. The thorough understanding of EM precursors in terms of physics is a path to achieve deeper knowledge of the last stages of the EQ preparation process, and thus a path to more credible short-term EQ prediction. A seismic shift in thinking towards basic science will lead to a renaissance of strict definitions and systematic experiments in the field of EQ prediction. Kossobokov (2006) emphasizes that "no scientific prediction is possible without exact definition of the anticipated phenomenon and the rules, which define clearly in advance of it whether the prediction is confirmed or not."

As noted above, there may be legitimate reasons for the critical views concerning the reliability of EM precursors. These negative views are supported by the fact that specific "puzzling features" are systematically observed in candidate pre-seismic EME. Specifically, (i) EM silence in all frequency bands appears before the main seismic shock occurrence, and (ii) EM silence is also observed during the aftershock period.
In this work we focus on this point. We examine whether the aforementioned features of EM silence are really puzzling ones or, instead, reflect well-documented characteristic features of fracture process.

The main concept is to investigate whether laboratory experiments reproduce these "puzzling features". It was suggested in the earlier literature that "the mechanism of EQs is apparently some sort of laboratory fracture process" (Mogi, 1962a, b, 1968, 1985; Ohnaka, 1983; Ohnaka and Mogi, 1982; Scholz, 1968, 1990; Scholz et al., 1973; Sobolev, 1995; Ponomarev et al., 1997; Ohnaka and Shen, 1999; Sobolev and Ponomarev, 2003; Muto et al., 2007; Kuksenko et al., 1996, 2005, 2007, 2009; Lei and Satoh, 2007). Recently, the aspect of self-affine nature of faulting and fracture is widely documented from analyses of data from both field observations and laboratory experiments in the spatial, temporal, and energy domains (Mandelbrot, 1982; Mandelbrot et al., 1984; Zavyalov and Sobolev, 1988; Gabrielov et al., 1999; Kuksenko et al., 2007; Davidsen and Schuster, 2002; Lockner et al., 1992; Diodati et al., 1991; Kapiris et al., 2004). A number of studies indicate a similarity of the statistical properties of laboratory seismicity in terms of $\mathrm{AE}$ and $\mathrm{EME}$ on the one hand, and seismicity on the geological scale on the other hand, in the spatial, temporal, and energy domains. Therefore, we have the opportunity to ask the following important question: Can we identify any accordance between the lab and the field by means of the two above-mentioned puzzling features?

Of course, it is a risky practice to extend findings rooted in laboratory experiments to the geophysical scale. However, one cannot ignore the comparison between failure precursors at the laboratory and geophysical scales, especially taking into account the ubiquitous power law in the fracture process, i.e., self-similarity between fracture patterns on various scales.

The remainder of this article is organized as follows. Section 2 presents the up-to-date theoretical and laboratory background; this includes a brief description of the two-stage model for the EQ generation process and the percolation theory of fracture, upon which the presented analysis is based. Based on the evidence highlighted in Sect. 2, an explanation of the pre-seismic $\mathrm{MHz}$ and $\mathrm{kHz}$ EME silence is attempted in Sects. 3 and 4, respectively. In Sect. 5, the silence observed sequentially in all EM bands is interpreted from the percolation theory of fracture point of view. Finally, the results are discussed and summarized in Sect. 6.

\section{The up-to-date theoretical and laboratory background}

In this section, the information relative to the fractureinduced $\mathrm{MHz}-\mathrm{kHz}$ EME that is available from the up-to-date published theoretical as well as laboratory works is synthetically presented. Based on the evidence highlighted in this 
section, an explanation of the silence feature observed in the $\mathrm{MHz}-\mathrm{kHz}$ EM precursory signals is attempted in Sects. 3, 4, and 5 .

\subsection{Two-stage model of EQ dynamics by means of MHz-kHz EM emissions}

Based on the concepts presented in the Introduction, we have studied the possible seismogenic origin of the $\mathrm{MHz}-\mathrm{kHz}$ anomalies recorded prior to significant shallow EQs that occurred on land, trying to answer three fundamental questions: (i) how can we recognize an EM observation as a pre-seismic one? (ii) How can we link an individual EM precursor with a distinctive stage of the EQ preparation process? and (iii) How can we identify precursory symptoms in EM observations that indicate that the occurrence of the EQ is unavoidable? The second question is the fundamental one. The answers to the other two questions should be rooted in the answer of the second one. EM phenomena associated with EQs may be recognized as real EQ precursors only when the physical mechanism of their origin is clarified (Pulinets and Boyarchuk, 2004; Cicerone et al., 2009).

The basic information that guided our thinking was the following well-established evidence. An important feature, observed both at laboratory and geophysical scale, is that fracture-induced $\mathrm{MHz}$ radiation consistently precedes the $\mathrm{kHz}$ radiation, indicating that these two emissions correspond to different characteristic stages of the fracture/EQ preparation process (Kumar and Misra, 2007; Qian et al., 1994; Eftaxias et al., 2002, and references therein; Baddari and Frolov, 2010). It is believed that the crack size varies in inverse proportion to the carrier frequency. Therefore, the transition from the $\mathrm{MHz}$ to $\mathrm{kHz}$ EME signals the hierarchical development of failure, namely the transition from smaller to larger cracks. As is consistent with this, laboratory experiments in the context of mesomechanics support the concept that the $\mathrm{MHz}$ and $\mathrm{kHz} \mathrm{EM}$ modes consecutively observed at the geophysical scale may signalize the transition of plastic flow localization from the mesoscale to the macroscale, culminating in global fracture (Eftaxias et al., 2007). Our studies have verified that the $\mathrm{MHz}$ and $\mathrm{kHz}$ EM physical fields refer to completely different fracture mechanisms. More precisely, we have introduced the following two-stage model (Fig. 1) (Kapiris et al., 2004; Contoyiannis et al., 2005; Contoyiannis and Eftaxias, 2008; Papadimitriou et al., 2008; Eftaxias et al., 2007, 2010; Eftaxias, 2012, and references therein):

1. The initially emerging $\mathrm{MHz}$ EM field originates from the cracking in the highly heterogeneous material that surrounds the backbone of asperities, distributed along the stressed fault sustaining the system.

2. The abrupt emergence of strong avalanche-like $\mathrm{kHz}$ EME is thought to be due to the fracture of the family of the asperities themselves.

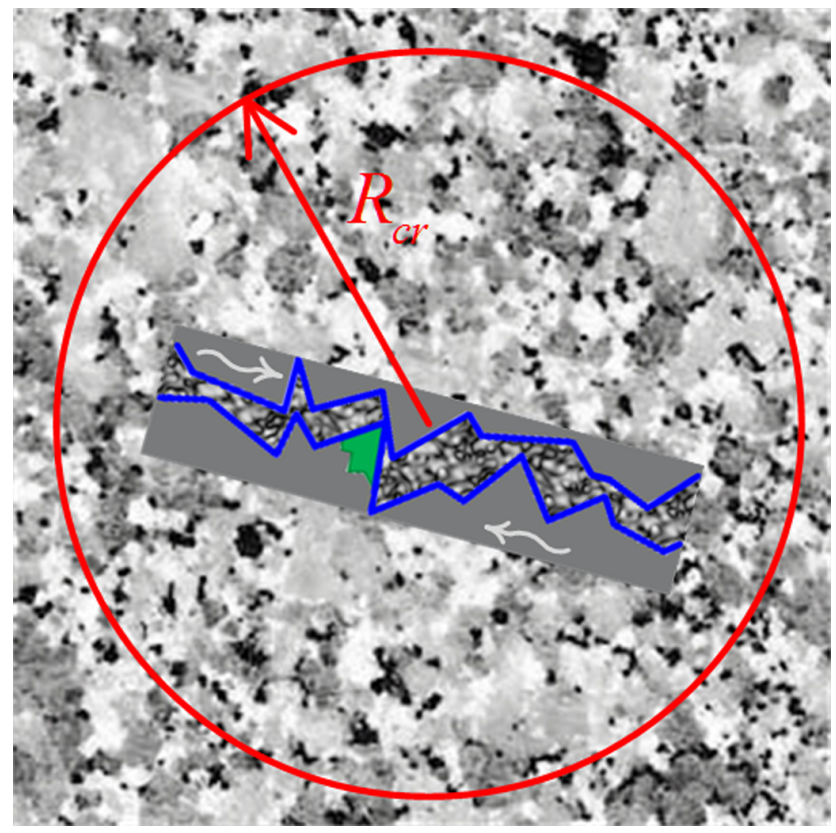

Fig. 1. A fault (blue lines) is embedded in an heterogeneous environment. The EQ preparation process at the first stage concerns an area of $R_{\mathrm{cr}}$ radius around the fault where the heterogeneous media takes part in the fracture process, emitting MHz EME during the cracking of heterogeneous media. The symmetry breaking signals the transition from the phase of non-directional, almost symmetrical, cracking distribution to a directional localized cracking zone along the direction of the fault. The EQ is inevitable if and only when the asperities break (green highlighted area), emitting the $\mathrm{kHz}$ EME during the second stage, and then an EME silence follows.

\subsubsection{Focus on MHz EM field}

As an analogy to the study of critical phase transitions in statistical physics, it has been argued that the fracture of heterogeneous materials could be viewed as a critical phenomenon either at the laboratory scale or at the geophysical scale (Chelidze, 1982; Allegre et al., 1982; Sornette and Sornette, 1990; Herrmann and Roux, 1990; Sornette and Sammis, 1995; Sornette and Andersen, 1998; Bowman et al., 1998; Kossobokov et al., 1999; Sornette, 2000; Moreno, 2000; Gluzman and Sornette, 2001; Guarino et al., 2002; Rundle et al., 2003, and references therein). We note that other researchers have associated brittle rupture with a firstorder phase transition (Buchel and Sethna, 1997; Kun and Herrmann, 1999; Rundle et al., 2003, and references therein). However, this is still an open question. We have shown that the dynamics of the $\mathrm{MHz}$ EM emissions are characterized by antipersistency, namely a negative feedback mechanism that "kicks" the crack-opening rate away from extremes, while this mechanism is rooted in the heterogeneity of the fractured system (Eftaxias et al., 2003, 2009; Kapiris et al., 2004; Contoyiannis et al., 2005). This evidence guided us to examine whether the observed $\mathrm{MHz}$ EM precursor can 
be described in analogy with a thermal, continuous, secondorder transition in equilibrium. Recently, we have shown that this really happens (Contoyiannis et al., 2005). Moreover, combining ideas of Levy statistics, non-extensive Tsallis statistical mechanics, and criticality with features hidden in the precursory $\mathrm{MHz}$ time series, we have shown that a truncated Levy-walk-type mechanism can organize the heterogeneous system to criticality (Contoyiannis and Eftaxias, 2008). We underline that the $\mathrm{MHz} \mathrm{EM}$ anomaly includes the crucial feature of "symmetry breaking" of critical phenomena. This feature permits the real-time, step-by-step monitoring of the damage evolution of the heterogeneous component in the focal area during mechanical loading. Indeed, our analysis reveals the following consecutive rupture process epochs as the EQ approaches (Contoyiannis et al., 2005; Eftaxias, 2012):

i. The critical epoch (critical window) during which the short-range correlations between the opening cracks evolve to long-range ones.

ii. The epoch of the "symmetry breaking" occurrence, namely the transition from the phase of nondirectional, almost symmetrical, cracking distribution to a directional localized cracking zone.

iii. The completion of the "symmetry breaking". The rupture process has been obstructed at the boundary of the backbone of strong asperities. We have proposed that the appearance of a MHz EM anomaly due to its nature is a necessary but not a sufficient requirement for the EQ occurrence. The completion of "symmetry breaking" simply means that the "siege" of asperities has already been started; that is, the applied stresses have already been focused on them. The abrupt emergence of strong avalanche-like $\mathrm{kHz}$ EME activity reveals the fracture of asperities if and when the local stress exceeds their fracture stress.

The aforementioned results not only clearly discriminate the recorded $\mathrm{MHz}$ anomalies from the background EM noise but are also consistent with the endorsement of the MHz EM anomaly as reflection of an underlying fracture process of a heterogeneous medium.

\subsubsection{Focus on kHz EM field}

The notably crucial character of the suggestion that the abrupt emergence of strong avalanche-like kHz EME activity reveals the fracture of asperities distributed along the fault sustaining the system, implying that the occurrence of the ensuing EQ is unavoidable as soon as $\mathrm{kHz}$ EMEs have been observed, requires strong support by well-established fundamental arguments.

\section{Arguments in terms of statistical analysis}

First, based on a multidisciplinary statistical analysis, we have shown that the $\mathrm{kHz}$ EM time series is characterized by the following crucial symptoms of an extreme phenomenon (Potirakis et al., 2011, 2012a, b, c; Eftaxias, 2012, and references therein): (i) high organization or high information content. Tools of information theory, concepts of entropy rooted in the extensive and non-extensive Tsallis statistical mechanics, and measures of complexity have been used in order to identify the aforementioned features (Shannon nblock entropy, Shannon n-block entropy per letter, conditional entropy, entropy of the source, Kolmogorov-Sinai entropy, T entropy, approximate entropy - ApEn, Fisher information, correlation dimension, R/S analysis, detrended fluctuation analysis, fractal dimension, and finally fractal wavelet spectral analysis). (ii) Strong persistency, indicating the presence of a positive feedback mechanism in the underlying fracto-EM mechanism that leads the systems out of equilibrium. (iii) Absence of any footprint of a second-order transition in equilibrium or truncated Levy-walk-type mechanism. (iv) Existence of a clearly preferred direction of fracture activities (cf. Fig. 2) (Potirakis et al., 2012b). We note that, according to the description of laboratory experiments and seismicity observations, AE and seismicity hypocenters tend to concentrate close to the nodal plane of a future main rupture (Mogi, 1968; Scholz, 1968; Ponomarev et al., 1997). The aforementioned results are consistent with the endorsement of the $\mathrm{kHz}$ EM anomaly as reflection of an underlying extreme event.

\section{Arguments in terms of universal structural patterns of the fracture process}

Nevertheless, from our point of view, even the aforementioned multidisciplinary statistical analysis per se does not link the $\mathrm{kHz}$ EM phenomenon with the fracture of asperities. The above-noted statistical results are likely to offer necessary but not sufficient criteria in order to recognize a $\mathrm{kHz}$ EM anomaly as an indicator of the fracture of asperities. The crucial question was whether different approaches could provide additional information that would allow for one to accept that the $\mathrm{kHz}$ EM anomalies signal the fracture of asperities. The basic information that guided our thinking was the following well-established evidence: despite the complexity of the fracture process, there are universally holding scaling relations. The aspect of self-affine nature of faulting and fracture is widely documented (Mandelbrot, 1982; Huang and Turcotte, 1988; Turcotte, 1997; Sornette, 2000). From our point of view, universal structural patterns of the fracture process should be included in a precursor associated with the fracture of asperities.

We have shown that the above-mentioned requirement is satisfied. Indeed, the following can be referenced:

i. Huang and Turcotte (1988) have stated that the statistics of regional seismicity could be merely a macroscopic reflection of the physical processes in EQ sources, namely the activation of a single fault is a reduced self-affine image of regional seismicity. In this 


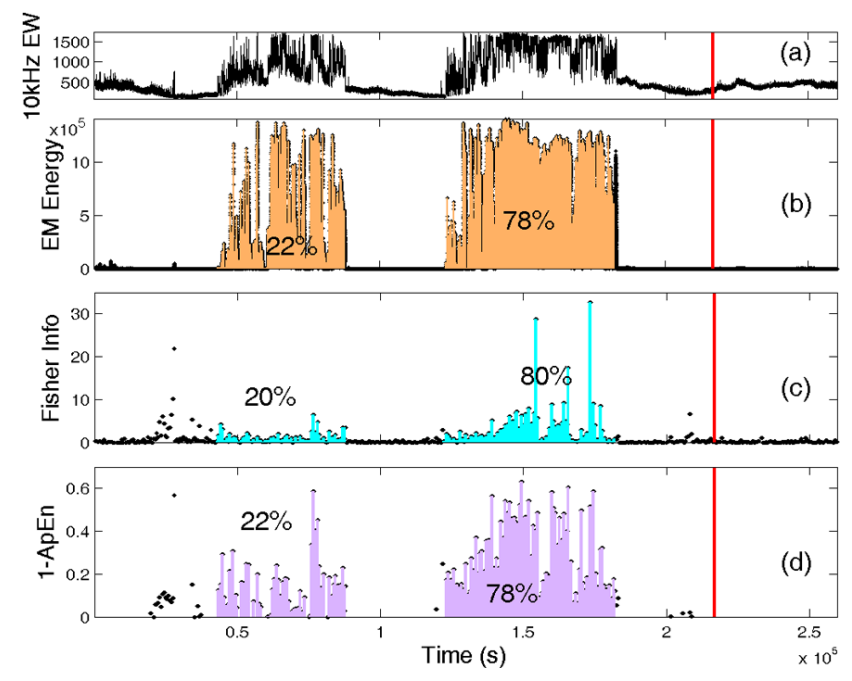

Fig. 2. (a) The two strong impulsive bursts in the tail of the recorded pre-seismic $\mathrm{kHz}$ EM emission prior to the Athens (Greece) EQ $(M=5.9)$ that occurred on 7 September 1999 (please refer to Fig. 1 in Papadimitriou et al., 2008). For the specific signal excerpt, the EM energy (b), the Fisher information (c), and approximate entropy (d) evolutions with time are presented. The colored areas indicate the energy, information, and 1-ApEn corresponding to the two bursts. The first (left) burst is responsible for $22 \%$ of the EM energy, $20 \%$ of the Fisher information, and $22 \%$ of the ApEn, while the second (right) for 78, 80, and 78\%, respectively. All graphs are time aligned for direct reference. The time of the EQ occurrence is indicated by the thick vertical red line (Fig 1. in Potirakis et al., 2012b).

direction, we have shown that the populations of the EQs that precede a significant seismic event and occur around its epicenter, and the "fracto-EM EQs" that emerge during the fracture of the population of asperities follow the same statistics, namely the relative cumulative number of EQs/EM-EQs vs. magnitude, either in terms of the traditional Gutenberg-Richter law (Kapiris et al., 2004) or by means of a model of EQ dynamics that is based on first principles of non-extensive statistical mechanics (Papadimitriou et al., 2008; Minadakis et al., 2012a, b). Furthermore, following the above-noted procedure, we have further shown that the activation of a single fault is a magnified self-affine image of laboratory seismicity as is observed by AE or EME (Kapiris et al., 2004).

ii. Fracture surfaces have been found to be self-affine following the persistent fractional Brownian motion $(\mathrm{fBm})$ model over a wide range of length scales (Chakrabarti and Benguigui, 1998). It has been proved that the profile of the observed $\mathrm{kHz}$ EMEs follows this model (Contoyiannis et al., 2005; Minadakis et al., 2012a). iii. The roughness of fracture surfaces is quantitatively characterized by the Hurst exponent, $H$, since the average height difference $\langle y(x)-y(x+L)\rangle$ between two points on a profile increases as a function of their separation, $L$, like $L^{H}$ with $H \sim 0.75$, weakly dependent on the nature of the material, on the failure mode, and on the spatial scale of fracture (Hansen and Schmittbuhl, 2003; Mourot et al., 2006). We have shown that the roughness of the profile of the observed $\mathrm{kHz}$ EM anomaly is consistent with the aforementioned universal $H$ value (Kapiris et al., 2004; Contoyiannis et al., 2005; Minadakis et al., 2012a).

\subsection{Are the AE and EME two sides of the same coin?}

The $\mathrm{kHz}$ EM emission is consistently launched in the tail of the fracture-induced EME both at the laboratory and the geophysical scale. Its absence during the EQ occurrence constitutes the most enigmatic feature in the study of EM precursors because older considerations had led to the erroneous conclusion that there is a contradiction between the laboratory and the field observed fracture-induced EME concerning the observed $\mathrm{kHz}$ EM silence. Indeed, the view up to a few years ago was that the observed $\mathrm{AE}$ and EME are two sides of the same coin. On the other hand, laboratory experiments in terms of $\mathrm{AE}$ were showing that this emission continues increasingly up to the time of the final collapse (Baddari and Frolov, 2010; Baddari et al., 2011; Schiavi et al., 2011). The combination of the above-mentioned information led to the erroneous conclusion that the appearance of $\mathrm{kHz} \mathrm{EM}$ silence in field observations just before the EQ occurrence is a puzzling feature in terms of laboratory experiments.

However, recent accumulated laboratory evidence indicates that the recorded $\mathrm{AE}$ and EME, in general, are not two sides of the same coin. There are two categories of $\mathrm{AE}$ signals - namely AE signals that are associated with EME signals, and $\mathrm{AE}$ signals that are not associated with EME signals (e.g., Yamada et al., 1989; Rabinovitch et al., 1995; Morgounov, 2001; Mori et al., 1994, 2004a, b, 2006, 2009; Mori and Obata, 2008; Lacidogna et al., 2010; Baddari and Frolov, 2010; Baddari et al., 2011; Carpinteri, 2012). Importantly, laboratory experiments performed recently reveal that the final stage of the failure process coincided in time with the maximum of $\mathrm{AE}$ and quiescence in $\mathrm{EME}$, while strong avalanche-like EME precedes this phase (Morgounov, 2001; Baddari et al., 2011). Therefore, the kHz EM silence just before the final collapse is observed both at laboratory and geophysical scale.

Baddari et al. (2011) emphasize that the alternating acoustic and $\mathrm{EM}$ quiescence in the $\mathrm{kHz}$ band was recorded during several different phases of block deformation, and not only during the last phase of failure process; the great decrease of EME was accompanied by an increase of $\mathrm{AE}$ intensity and vice versa. This may indicate the alternation between two different mechanisms involved in a fracture process. The 
aforementioned laboratory results strongly support the hypothesis that, in general, EME and $\mathrm{AE}$ represent different phases of the destruction process, and the combination of both emissions is important for the study of failure kinetics.

In the following we attempt to explain the reason that leads to the existence of the above-mentioned two categories of $\mathrm{AE}$ signals, namely the $\mathrm{AE}$ associated with EME and the AE not associated with EME. We attempt this explanation in terms of (i) the possible mechanism of generation of the observed fracture-induced EME and (ii) the dependence of the sensitivity of the emitted EME to the geometrical mode of the opening crack (shear or tensile), the brittleness index, Young modulus, and strength index of the material. We will see that the observed $\mathrm{AE}$ and EME are two sides of the same coin during the opening of tensile cracks in a material that is characterized by high brittleness index, Young modulus, and strength index. On the contrary, the phases of failure kinetics that are mainly characterized by frictionalnoise-type rearrangements due to the frictional slip between the pre-formed fracture structures can be accompanied only by acoustic (EQ) events, and not by EM events (Mori et al., 1994, 2004a, b; Mori and Obata, 2008). Subsequently, we try to tally-correspond the two phases of the emerged strong $\mathrm{kHz}$ EME and the $\mathrm{kHz}$ EME silence to two distinct, last phases of the EQ preparation process, namely to the fracture of entities of high index of strength, bulk modulus, and brittleness via the opening of tensile cracks on the one hand, and the frictional-noise-type rearrangements due to the frictional slip between the pre-formed fracture structures that provide a bearing-like "lubrication" mechanism between fault plates on the other hand.

\subsubsection{On the origin of the fracture-induced EME}

Several atomic models have been put forth to explain the origin of EM emission (Bahat et al., 2002, and references therein). Consider in particular the "movement of charged crack surfaces" (see Sect. II in Contoyiannis et al., 2005, and references therein). In this model, when a crack opens, the rupture of interatomic bonds leads to intense charge separation (Langford et al., 1987; Cress et al., 1987). Direct evidence of surface charges of opposite polarity on fresh fracture surfaces has been provided by Wolibrandt et al. (1983). Simultaneous measurements of the electron, ion, and photon emission (fracto-emission) accompanying fracture support the hypothesis that charge separation accompanies the formation of fracture surfaces (Langford et al., 1987; Dickinson et al., 1998; Gonzalez and Pantano, 1990; Miura and Nakayama, 2000; Mizuno and Mizuno, 2002). On the faces of a newly created crack, the electric charges constitute an electric dipole or a multi-pole of higher order, and, due to the crack wall motion, EM radiation is emitted (Miroshnichenko and Kuksenko, 1980; Khatiashvili, 1984; O'Keefe and Thiel, 1995; Molchanov and Hayakawa, 1995; Koktavy et al., 2004). Crack motion in fracture dynamics has recently been shown to be governed by a dynamical instability, which causes oscillations in the crack velocity and structure on the fracture surface. Evidence indicates that the instability mechanism is locally branching; that is, a multi-crack state is formed by repetitive, frustrated micro-fracturing events (Marder and Fineberg, 1996; Sharon and Fineberg, 1999). Laboratory experiments show intense EM fracto-emission during this unstable crack growth (Gonzalez and Pantano, 1990). In this unstable stage we regard the EME from the correlated population of opening cracks as a precursor of the final global instability (Contoyiannis et al., 2005).

If the above-mentioned interpretation is correct, the production of new fresh surfaces/rupture of bonds, due to cracking in the material, constitutes a necessary condition for the generation of EME. Mori and colleagues (Mori et al., 1994, 2004a, b; Mori and Obata, 2008) emphasize that the AE signals that are associated with EME signals are generated due to the creation of new fresh surfaces; on the contrary, AE signals that are not associated with EME signals are rooted in frictional noises due to the contact and/or the frictional slip/rolling between the pre-formed fracture surfaces.

\subsubsection{On the sensitivity of the fracture-induced EM anomaly to the geometrical mode of opening cracks}

Cracks can appear in solids under stress mainly in two different geometrical modes: tensile and shear. In the tensile mode, the displacements during the opening of cracks are perpendicular to the fracture propagation. Conversely, in the shear mode the displacements are in the same direction as those of the crack propagation. Due to the fact that the tensile fracture mode leads to larger distances between the surface charges compared to the shear mode, it has been proposed that an opening tensile crack behaves as a more efficient EM emitter (e.g., Yamada et al., 1989; Mori and Obata, 2008; Lacidogna et al., 2009, 2010, 2011; Baddari et al., 2011). Of course, the shear source EME should not be omitted; a certain separation between oppositely charged surfaces is guaranteed even in the shear fracture. However, the EME is more sensitive to the generation of tensile cracks than shear cracks (Molchanov and Hayakawa, 1995).

\subsubsection{On the sensitivity of the fracture-induced EM anomaly to elastic moduli, index of brittleness, and index of strength}

Elastic moduli, index of brittleness, and index of strength constitute crucial parameters for the detection of AE or EME from the material experiencing "damage". It was found that an increase of Young modulus, and index of brittleness and strength enhances the AE or EME amplitude (Hudson et al., 1972; Gol'd et al., 1975; Nitsan, 1977; Khatiashvili, 1984; Carpinteri, 1986, 1989, 1990, 1994; Frid et al., 1999, 2000; Rabinovitch et al., 2002; Fukui et al., 2005). On the other 
hand, accumulated experimental and theoretical evidence indicates that the elastic modulus significantly decreases as damage increases, nearing zero as the global fracture approaches (Lockner and Madden 1991; Guéguen et al., 1997; Lin et al., 2004; Shen and Li, 2004; Amitrano and Helmstetter, 2006; Chen, 2012; Chelidze et al., 1988, 1990).

Characteristically, a very important class of models of material failure is the fiber bundle models (FBMs), which capture the most important aspects of material damage. In the context of the discrete FBM, the stored elastic energy, $e_{\mathrm{f}}(t)$, in a single fiber at the time of failure is given by the formula

$e_{\mathrm{f}}(t)=V_{\mathrm{f}} E_{0} \varepsilon_{\mathrm{f}}^{2}(t) / 2$,

where $V_{\mathrm{f}}$ is the volume of the fiber, $E_{0}$ is the Young modulus of the fiber, and $\varepsilon_{\mathrm{f}}(t)$ the strain in the fiber at time $t$ (Turcotte et al., 2003). Consequently, the elastic energy release should be greatly reduced during the softening mode. Laboratory studies show that EM energy release rate has a direct correlation with the elastic strain release rate (Kumar and Misra, 2007; Chauhan and Ashok Misra, 2008). Therefore, it is reasonable to assume that when an element fails during the softening period, the emitted EM radiation is also greatly reduced. In summary, the sudden drop of stress just before the shock occurrence of its own accord signals a corresponding abrupt decrease in the amount of the elastic/fracto-EM energy that can be released, and finally provides a possible explanation of the observed EME gap.

\subsection{Fracture-induced EM anomalies in terms of percolation theory}

In the following, we investigate the behavior of the elastic modulus of heterogeneous media during the fracture process and the way that this influences the emission rate of EM radiation in terms of percolation theory. Moreover, in the context of this theory, we examine the discrimination of different thresholds of concentration of defects in a medium. The first of them could correspond to transfer phenomena and the subsequent two of them to different phases of fracture.

\subsubsection{On the dependence of the fracture-induced emission pattern on the elastic modulus}

It has been shown that the problem of fracture of heterogeneous media corresponds mathematically to problems of the percolation theory, which describe quantitatively the connectivity of components in a non-homogeneous system (Chelidze, 1979, 1980a, b, 1982, 1986, 1987, 1993; Chelidze at al., 2006; Arbadi and Sahimi 1990). It appears that the elastic modulus, $M$, decreases from the initial value for an intact lattice over nearly five orders of magnitude according to the expression $M \propto M_{0}\left(x-x_{\mathrm{cr}}\right)^{-3.6}$, where $x$ is the current concentration of breakages and $x_{\mathrm{cr}}$ is the percolation threshold for a given finite system; it has been found that $x_{\mathrm{cr}}=0.494$. Arbadi and Sahimi (1990) have studied models of mechanical breakdown in disordered solids by large-scale Monte Carlo simulations. Their results also show that the Young's modulus $Y$ reduces to zero well before the total breaking of bonds, namely when the fraction $p$ of the unbroken bonds is approximately 0.2 (see Fig. 1 in Arbadi and Sahimi, 1990). A model of elastic wave emission and amplitude distribution during the failure has been developed (Chelidze and Kolesnikov, 1984; Chelidze 1993) that assumes that the emergence of a new defect is associated with an emission event and that the (conventional) emission amplitude $A_{\mathrm{c}}$ generated by each elementary event depends directly on the increment of the size of the resulting defect cluster, induced by this single event,

$A_{\mathrm{c}}=A_{0}\left(\left(\sum_{i=1}^{k+1} s_{i}\right)^{2}-\sum_{i=1}^{k} s_{i}^{2}\right)^{1 / 2}$,

where $k$ is the number of clusters linked by the elementary defects, $s_{i}$ is the number of sites in the $i$-th merging cluster, and $A_{0}$ is a conventional amplitude generated by the nucleation act of a single isolated defect. According to Eq. (2) the emission amplitude should increase with approach to the percolation threshold as merging of large clusters generates a larger dynamical event. Numerical experiments show that the amplitude spectrum of the quantity $A_{\mathrm{c}}$ possesses basic characteristics of the $\mathrm{AE}$ of fracture, namely the increase in mean amplitude, and the growth of the amplitude dispersion as the percolation threshold is approached (Chelidze, 1986, 1993). On the other hand, in $A_{0}$ we take into account the current elastic modulus $M$ of the system, so that $A_{0}=f(M)$, where $M \propto\left(p-p_{\mathrm{c}}\right)^{\alpha}$; that is, $M$ and correspondingly $A_{0}$ also change with increase of damage $p$ - namely $M$ decreases according to the power law of Chelidze (1993) and $p_{\mathrm{c}}$ is the corresponding percolation threshold. Physical experiments involving artificial sequential damaging of the plastic lattice confirm the validity of the expression $M \propto\left(p-p_{\mathrm{c}}\right)^{\alpha}$ with $a=-3.6$ (Chelidze et al., 1990).

A theoretical expression for energy emission, $\Delta E$, at the addition of one damaged site, expressed by means of fundamental percolation functions, is given in Chelidze (1986), where $\Delta E$ is calculated as a derivative of the mean size of finite clusters, $s$ :

$$
\begin{aligned}
\Delta E & =\frac{\mathrm{d}}{\mathrm{d} p} \sum_{s} s^{2} n_{s}(p) / \sum_{s} s n_{s}(p)=p \frac{\mathrm{d} s(p)}{\mathrm{d} p}+s(p) \\
& =\left(\sum_{i=1}^{k+1} s_{i}\right)^{2}-\left(\sum_{i=1}^{k} s_{i}^{2}\right),
\end{aligned}
$$

where $n_{s}$ is the number of clusters of size $s$, and differentiating we take into account that the sum $\sum_{s} s n_{s}(p)=p$ for $p<p_{\mathrm{c}}$.

$$
\begin{aligned}
& \text { For } p \rightarrow p_{\mathrm{c}} \text { we get } \\
& \Delta E \approx\left|p-p_{\mathrm{c}}\right|^{-(\gamma+1)}=s\left(1+L_{\mathrm{R}}\right),
\end{aligned}
$$


where $L_{\mathrm{R}}$ is the average number of cutting ("red", single) bonds in an incipient infinite cluster (IC) (Chelidze and Kolesnikov 1984). The same approach has been used by Sammis and Sornette (2002), and they obtained similar expressions for $\Delta E$. In the above treatment the elastic modulus $M$ of the system was considered as a constant, and thus it was ignored. This is true on the first stage of fracture process when the cracks form relatively small finite clusters, which can be considered as isolated soft inclusions in the (globally) rigid medium. However, when approaching the percolation threshold the elastic modulus depends on the concentration of defects $p$.

So the expression for emitted energy for $p \rightarrow p_{\mathrm{c}}$, taking into account that $M$ is a function of $p$, can be written as

$$
\begin{aligned}
\Delta E & \approx \frac{\mathrm{d} M}{\mathrm{~d} p}\left|p-p_{\mathrm{c}}\right|^{-(\gamma+1)} \approx \alpha\left|p-p_{\mathrm{c}}\right|^{\alpha-1}\left|p-p_{\mathrm{c}}\right|^{-(\gamma+1)} \\
& \approx \alpha\left|p-p_{\mathrm{c}}\right|^{\alpha-\gamma-2} .
\end{aligned}
$$

Equation (5) clarifies that the drastic decrease of elastic modulus in the last stage of fracture of a heterogeneous medium can significantly change the emission pattern described by Eq. (2), because the "standard amplitude" $A_{0}$, which includes the elastic characteristics of media, should also diminish when approaching the percolation threshold. Then, if the decrease of $A_{0}$ dominates over the increase of the second (right-hand) factor of Eq. (2) during the final stage of fracture, the mean amplitude of emission can even decrease with defect proliferation. This can cause apparent silence before the mechanical collapse.

\subsubsection{On the discrimination of thresholds for transport and fracture in terms of percolation theory}

The discrimination of thresholds for transport and fracture in terms of percolation theory also offers a quantitative explanation of the observed EM silence in various frequency bands. A percolation model, which considers the fracture of heterogeneous media as a critical phenomenon, was suggested in 1978 by T. Chelidze (first publications 1979, 1980a, b, 1982), and has been developed in many subsequent works (see, for example, Herrmann and Roux, 1990). This model predicts the existence of "critical points" or percolation transitions in various properties of the material, when its characteristics change dramatically (Eftaxias and Chelidze, 2005). These transitions (metal-dielectric, gelsol, permeable-impermeable, consolidated-unconsolidated) are observed for all the so-called generalized conductivities or transport-related properties of materials, which critically depend on the connectivity of transport channels. In many cases, the percolation thresholds for various properties are the same, which means that the corresponding transport processes have the same geometrical basis. At the same time it is evident that the transition impermeable-permeable does not mean that the three-dimensional (3-D) systems become mechanically unconsolidated. The transition consolidatedunconsolidated, which allows for the division of the 3-D material into at least two parts demands much larger concentration of voids. Consequently, the percolation model of fracture is associated with two different thresholds of concentration of defects, $x$, namely

i. the "hydraulic threshold", $x_{\mathrm{c}}$, and

ii. the "mechanical or damage threshold", $x_{\mathrm{m}}$, where the infinite cluster (IC) is formed, and the solid disintegrates (Chelidze, 1986).

As a rule, the relation $x_{\mathrm{m}}>x_{\mathrm{c}}$ holds.

The EQ is considered as a shear displacement along the fault plane. However, a layer of thickness $h$ of the material should contain the IC. Chelidze (1986) has proposed that the associated problem of shear displacement along the fault can be formulated in terms of mechanical percolation as following: "find the concentration $x_{\mathrm{mf}}$ at which the flat infinite cluster (FIC) of voids (cracks) spanning the layer of thickness $h$ would be formed in a 3-D body". The problem of such an FIC has been solved in the percolation theory (Shklovsky, 1984; Chelidze, 1987). The required critical concentration for the formation of FIC, $x_{\mathrm{mf}}$, is (Chelidze, 1986)

$x_{\mathrm{mf}}=x_{\mathrm{m}}\left[1+d\left(r_{\mathrm{s}} / h\right)^{1 / \nu_{3}}\right]>x_{\mathrm{m}}$,

where $r_{\mathrm{S}}$ is the mean distance between defects, $d$ is a constant close to unity, and $\nu_{3}=0.9$ for 3 -D. The FIC mechanical percolation model is very similar to the well-known physical models of seismic process evolution, namely dilatancydiffusion and avalanche fracturing models (Mjachkin et al., 1975; Scholz et al, 1973). Based on the above-mentioned considerations, it might be concluded that many transport properties are activated before the collapse of the sample (Paterson, 1978; Chelidze, 1986). We note that the hydraulic percolation thresholds correspond to a critical crack density of about 0.1 , while experimental data show that the mechanical threshold is on the order of 1 (Zhang et al., 1994a, b).

\section{An explanation of the observed pre-seismic $\mathrm{MHz}$ EME silence}

In the following, we attempt an explanation of the observed pre-seismic MHz EME silence in terms of the two-stage model, laboratory experimental evidence, and percolation theory as presented in Sect. 2, as well as in terms of the foreshock activity.

\subsection{Arguments by means of the two-stage model of EQ generation}

The consistently observed pre-seismic silence of the $\mathrm{MHz}$ EME is fully understood in the context of the abovementioned two-stage model of EQ generation (see Sect. 2.1): 
this emission stops well before the EQ occurrence, namely when the fracture of the heterogeneous material that surrounds the backbone of asperities distributed along the stressed fault sustaining the system has been completed and the stresses build up up on the family of asperities; the preparing EQ will occur if and when the local stress exceeds the fracture stresses of these strong entities. Accumulated experimental and theoretical evidence support this proposal, as follows.

\subsection{Arguments by means of laboratory experiments}

Laboratory experiments by means of AE and EME strongly justify the appearance of $\mathrm{MHz}$ silence prior to the global failure; the $\mathrm{MHz}$ activities are rooted in earlier lower levels of fracture in comparison to that of the $\mathrm{kHz}$ activity (Eftaxias et al., 2002, and references therein; Baddari and Frolov, 2010). Experimental studies by means of mesomechanics (see Sect. 2.1) support the consideration that the MHz activities are launched earlier in comparison to the $\mathrm{kHz}$ activities (Eftaxias et al., 2007).

\subsection{Arguments by means of foreshock activity}

We recall that the precursory MHz EM phenomenon can be described in terms of a second-order thermal phase transition in equilibrium. It is reasonable to accept that such a phenomenon should be associated with a stage of the EQ preparation process that precedes the final stage of the fracture where the extreme seismic event is formed; it cannot originate from the final stage. Consequently, it might be deduced that the observed pre-seismic MHz EM silence before the seismic shock occurrence reflects a well-established precursory feature of the fracture process. However, the foreshock activity and the precursory fracture-induced MHz EM emission constitute two sides of the same coin. A question that logically arises is whether the foreshock activity during the last week before the main shock occurrence also behaves as a critical phenomenon. A recent analysis leads to a positive answer. Based on the recently introduced concept of natural time (Varotsos et al., 2011), we have proved that (Potirakis et al., 2013) (i) the foreshock seismic activity that occurs in the region around the epicenter of the oncoming significant shock from a few days up to one week before the main shock occurrence and (ii) the observed MHz EM precursor that emerges during the same period both behave as critical phenomena. This result strongly supports the seismogenic origin of the observed EM precursor, its behavior as a critical phenomenon, and finally the hypothesis that the observed $\mathrm{MHz}$ EM silence before the global instability occurrence is an expected and non-puzzling feature in the context of fracture processes.

In the following paragraph we attempt to explain the observed $\mathrm{MHz}$ EM silence based on the pioneering work of
Chelidze, who first studied the EQ preparation process by means of the percolation theory (Stauffer, 1985).

\subsection{Arguments by means of percolation theory}

In Sect. 2.3.1 it has been shown by means of fundamental percolation functions that the extreme decrease of elastic modulus when the percolation threshold is approaching diminishes the fractured-induced AE or EME, causing apparent silence before the mechanical collapse of the heterogeneous medium. We recall that laboratory experiments have shown that an increase of the elastic modulus enhances the AE or EME amplitude (see Sect. 2.2.3), while accumulated experimental and theoretical evidence indicate that the elastic modulus significantly decreases as damage increases, nearing zero as the global fracture approaches (see Sect. 2.2.3).

Based on this evidence, the appearance of silence in the $\mathrm{MHz}$ EM band before the EQ occurrence is an expected feature according to mechanical percolation theory.

\section{An explanation of the observed pre-seismic $\mathrm{kHz}$ EME silence}

Guided by the laboratory results presented in Sect. 2.2 - that (i) there are two categories of AE signals; (ii) an opening tensile crack behaves as a more efficient EM emitter than a shear crack; and (iii) the higher the brittleness index, strength index, and elastic modulus, the stronger the emitted EME we propose that the consecutively emerging strong impulsive $\mathrm{kHz}$ EME and $\mathrm{kHz}$ EM silence represent two different, distinct phases of the destruction process: the fracture of the asperities and the following frictional-noise-type mechanism phase that includes the occurrence of the main seismic event, respectively.

Note that laboratory studies support the aforementioned two-phase proposal. Characteristically, Zhang et al. (2009) have recently presented a physical theory for brittle failure that aims to explain both the phenomenological and microstructural observations. Based on experimental results, the authors conclude that "the localized failure process of rock experiences two stages: the brittle breakage stage (bond rupture) and the sliding stage (frictional resistance of failure plane mobilization)."

\subsection{Focus on the first phase}

In Sect. 2.1 we have reported accumulated evidence supporting the proposal that the observed $\mathrm{kHz}$ EME is rooted in the fracture of multi-asperity system distributed along the activated fault. The fracture of this system obeys all the requirements to form a population of efficient $\mathrm{kHz}$ EM radiations as they have been presented in Sect. 2.2. More precisely, the asperities are characterized by high index of brittleness, high index of strength, and high bulk modulus. We do expect, on mechanical grounds, that large EQs will nucleate on strong 
entities, which can store sufficient stress to produce a rupture that will not be easily stopped (Sornette, 1999, and references therein). On the other hand, the fracture of asperities happens through the opening of tensile cracks. Laboratory studies clearly suggest that the formation of the macroscopic fracture plane is more likely to be the result of a tensile than a shear process (Hallbauer et al., 1973).

Herein we present more evidence associating the observed pre-seismic kHz EME with the fracture of asperities. From experimental studies on the frictional movement between rock surfaces, it is known that when the static friction resistance is overcome, the frictional surfaces suddenly slip, lock, and then slip again in a repetitive manner (Matcharashvili et al., 2011). If one asperity fails, the stress is transferred to the adjacent element on which an induced failure could occur. The unstable transition from the static friction to kinetic one is known as "stick-slip" state; EQs have long been recognized as resulting from a stick-slip frictional instability (Scholz, 1998). Recent works reveal that stick-slip friction events observed in the laboratory and EQs in continental settings, even with large magnitudes, have similar rupture mechanisms (Lockner and Okubo, 1983; McGarr and Fletcher, 2003; McGarr et al., 2010). During the fracture of an asperity a $\mathrm{kHz}$ EM avalanche, a "fracto-EM EQ" is emitted. The avalanche-like feature of the observed $\mathrm{kHz}$ EME activity is consistent with the suggestion that the population of the observed "fracto-EM EQs" reveal the population of the fractured asperities. We note that the time scale of the final tertiary stage of creep is characterized by an avalanche in the strain rate (Morgounov, 2001) as well.

The fracture of a strong asperity should be accompanied by a sharp drop in stress. Indeed, laboratory studies suggest that the strong avalanche-like EM signals are generated only during sharp drops in stress, while the amplitude of the emitted EM fields is proportional to the stress rate (Fukui, 2005; Carpinteri et al., 2011, 2012; Lacidogna et al., 2011). These sharp drops are attributed to a rapid decay of the mechanical properties, generated by formation of new micro-cracks during the loading process (Carpinteri et al., 2012).

We reiterate that the pre-seismic $\mathrm{kHz}$ EME behaves as a fractal radiation (see Sect. 2.1.2). Muto et al. (2007) conducted a friction laboratory experiment simulating the motion of an asperity on a fault plane, and observed photon emissions due to electric discharges by dielectric breakdown of ambient gases from friction contact between rock minerals. Those authors conclude that the frictional discharge occurring at asperities on the fault plane can be one of the origins of fractal EM radiation observed prior to EQs.

\subsection{On the second phase (EM silence)}

The herein-examined crucial question is whether, within the context of materials science, mechanisms have been proposed that could explain the absence of $\mathrm{kHz}$ EM radiation just before the EQ occurrence. We argue that such mechanisms have been proposed, while recent laboratory and numerical experiments fully justify the hypothesis that the emerged silence is the last precursory feature of an imminent significant EQ and not a puzzling feature.

\subsubsection{From microscopic to macroscopic mechanics}

We propose that the resolution of the enigmatic feature under study may be hidden in the resolution of another wellknown paradox of EQ dynamics that the geophysicists call the heat-flow paradox (Anoosherhpoor and Brune, 1994; Alonso-Marroquin et al., 2006, and references therein). According to common sense, when two blocks grind against one another, there should be friction, and that should produce heat. However, measurements of heat flow during EQs are unable to detect the amount of heat predicted by simple frictional models. Calculations using the value of rock friction measured in the laboratory, i.e., a typical friction coefficient between 0.6 and 0.9 , lead to overestimation of the heat flux. As an example, one refers in this context to the heatflow observations made around the San Andreas fault, which show that the effective friction coefficient must be around 0.2 or even less (Alonso-Marroquin et al., 2006, and references therein).

Various relevant mechanisms have been proposed; however, the correct assessment of frictional heat production during shear is a central issue. There are different natural systems that fail through a localized failure in narrow shear zones. These so-called shear bands appear, for example, in granular packings (Astrom et al., 2000). Tectonic faults are a characteristic example of shear failure in narrow zones; many naturally occurring faults and shear zones contain regions of granular material. The gouge particles, i.e., fragmented rock inside the fault zone, play a fundamental role in influencing the macroscopic behavior of these systems (Latham et al., 2006; Welker and McNamara, 2011). The formation of rolling bearings inside the gouge has been introduced as a possible explanation for a substantial reduction of the effective friction coefficient. A simplified picture assumes that the gouge is filled with more or less round grains that, as the plates move, can roll on each other, thus reducing the amount of frictional dissipation. Granular dynamics simulations (Astrom et al., 2000; Mahmoodi Baram et al., 2004; Herrmann et al., 2004) have demonstrated the spontaneous formation of such bearings, while laboratory experiments also demonstrated the spontaneous formation of such bearings (Veje et al., 1999). We note that Wilson et al. (2005) have shown that gouge from the San Andreas fault, California, with $\sim 160 \mathrm{~km}$ slip, and the rupture zone of a recent EQ in a South African mine with only $\sim 0.4 \mathrm{~m}$ slip, display similar characteristics, in that ultrafine grains approach the nanometer scale, gouge surface areas approach $80 \mathrm{~m}^{2} \mathrm{~g}^{-1}$, and grain size distribution is non-fractal. Wilson et al. (2005) also propose that the observed fine-grain gouge is not related to quasi-static 
cumulative slip but is instead formed by dynamic rock pulverization during the propagation of a single $\mathrm{EQ}$.

The above-mentioned picture, which refers to the phase that follows the fracture of asperities, seems to justify the observed EM silence. As the plates move, the grains can roll on each other as rolling bearings, and thus the resulting deformation is not accompanied by significant fracturing, i.e., by formation of new fracture surfaces via the opening of tensile cracks. Such frictional-noise-type rearrangements due to the frictional slip between the pre-formed fracture structures can be accompanied only by acoustic (corresponding to EQ in geophysical scale) events and not by EM events (Mori et al., 1994, 2004a, b; Mori and Obata, 2008). As already noted in Sect. 2.2, laboratory experiments reveal that AE of maximal intensity are recorded directly before and during the fragmentation of the specimen, while strong avalanche-like EME precedes this phase (Baddari et al., 2011; Morgounov, 2001).

Numerical studies support the proposal that the final stage of the failure process is not accompanied by significant fracturing. Park and Song (2013) have presented a new numerical method for the determination of contact areas of a rock joint under normal and shear loads. They report that "after the peak stage, the contact area ratio decreased rapidly with increasing shear displacement, and few inactive elements came into contact until the residual stage. At the residual stage, only small fractions of $0.3 \%$ were involved in the contact".

As already noted, the efficiency of generating EME is thought to be smaller in shear cracks than in tensile cracks. Yamada et al. (1989) conclude that "the final main shock is considered to be a result of shear faulting, which may occur by connecting numerous cracks produced previously", while Stanchits et al. (2006) conclude that close to failure the relative contribution of tensile events decreased. A concomitant increase of double-couple events indicating shear suggests that shear cracks connect previously formed tensile cracks. Therefore, it is reasonable to expect that the anomalous EME will be observed only before an EQ but not during the main shock (Yamada et al., 1989). According to Benioff (1951), creep may be purely compressional, purely shearing, or a combination of two. In the last case, the compressional phase occurs first. Importantly, the author proposes that the shearing phase follows an interval of 0.01-2.4 days. This time window is in agreement with the observed duration of the kHz EM gap.

\section{Interpretation of the sequential appearance of the ULF-MHz-kHz precursors in terms of percolation theory of fracture}

It must be mentioned here that well-established direct current (DC) ultra low frequency (ULF) EM precursors $(<1 \mathrm{~Hz})$ have been observed to appear earlier than the $\mathrm{MHz}$ and $\mathrm{kHz}$ ones. Their lead time extends up to three months before the EQ occurrence (Varotsos, 2005; Molchanov and Hayakawa,
2008). In this section we try to explain why the DC-ULF, $\mathrm{MHz}$, and $\mathrm{kHz} \mathrm{EM}$ anomalies appear one after the other and thus why the DC-ULF EM precursors are also absent during the EQ occurrence. The relevant arguments are based on the discrimination of thresholds for transport and fracture in terms of percolation theory (see Sect. 2.3.2).

It is reasonable to assume that the various short-lived EM pre-seismic anomalies should be observed with the following order of time: (i) first, precursory phenomena associated with transport properties appear well before the collapse. (ii) Second, fracture-induced precursory phenomena associated with the formation of the IC are launched. The MHz EM activity is such a phenomenon. It might be considered that the precursory MHz EME, which is emitted during the last week before the shock occurrence, is associated with the mechanical threshold $x_{\mathrm{m}}$, namely with the formation of flat cluster of thickness $h$. During this formation, the fracture or grinding of weak heterogeneous material distributed around the main fault emits the antipersistent $\mathrm{MHz}$ radiation. Its behavior as a second-order phase transition as well as the observed symmetry breaking during the temporal evolution of this radiation strongly support the aforementioned hypothesis, especially the association of the $\mathrm{MHz}$ precursor with the formation of flat cluster. (iii) Third, the fracture-induced precursory phenomena rooted in the formation of the FIC emerge. The kHz EME is such a phenomenon. The observed persistent $\mathrm{kHz}$ EM activity from a few tens of hours up to a few minutes before the EQ occurrence might emerge around the critical mechanical threshold $x_{\mathrm{mf}}$, i.e., during the fracture of high strength and brittle asperities sustaining the system.

Different mechanisms for the generation of the first appearing DC-ULF precursors have been proposed, like the ones presented by Varotsos (2005) and Molchanov and Hayakawa (1995), while publications have also suggested that electrokinetic phenomena can provide the basis for the generation of ULF electrical and magnetic precursors (Mizutani et al., 1976; Surkov et al., 2002, and references therein).

Here we focus on the electrokinetic phenomena. Electrokinetic electric and magnetic fields result from fluid flow through the crust in the presence of an electric double layer that is formed at the solid-liquid interface. This electric double layer is made up of a layer of ions (the Helmholtz layer) anchored to the surface of the solid (e.g., the rock) and as a diffuse mobile layer of ions of opposite sign (the GouyChapman zone) that extends into the liquid phase. Fluid flow in this system transports the ions in the fluid in the direction of flow, and thus electric currents result. In this way, when a fluid flows through a porous medium, a potential will be developed across the length of the sample because of the relative motion between the solid and the liquid. Thus, it has been suggested that a fracture-induced precursory ULF streaming potential appears. The electrokinetic phenomenon, as a mechanism of generation of the ULF pre-seismic signals, should be associated with the "hydraulic threshold" within the context of the percolation model, because it is 
not observed in partially saturated disperse systems. A recent work (Surkov et al., 2002) strongly supports the abovementioned hypothesis. Electrokinetic effects possibly associated with the EQ preparation process have been theoretically investigated under the assumption that the structure of pore space around a hypocentral zone has fractal properties. This study verifies well-established experimental results - namely the predicted dependence of the electrical anomalies amplitude $E$ on the EQ magnitude, the weak values of magnetic variations at far distances, and the presence of highly conductive channel(s) between the source and observation in order for the precursory electrical anomalies to be detectable. We note that, as the electrokinetic processes are relatively slow, their contribution to higher frequency bands is negligible; the electrokinetic phenomena decay at high frequencies. On the other hand, direct EM emissions at high frequencies are not detectable at this stage of fracture, as the concentration of cracks at hydraulic threshold is very small, while their orientation is random and the emitted EM fields cancel each other.

\section{Discussion and conclusions}

In this work we studied a paradox associated with a series of different EM seismic precursors ranging from $\mathrm{DC}$ to $\mathrm{MHz}$ frequency bands, namely their observed silence before the main seismic shock occurrence. We conclude that the considered puzzling features of the $\mathrm{MHz}-\mathrm{kHz}$ fracture-induced EME are well-justified features and not paradoxical ones if we employ for their resolution a two-stage model for the EQ generation process and the percolation theory of fracture. Moreover, the silence of DC-ULF precursors is also explained based on percolation theory and the hypothesis that they are generated due to the electrokinetic phenomenon. Well-established concepts of the rupture process - in terms of universal structural patterns of the fracture process; recent laboratory experiments that prove that $\mathrm{AE}$ and $\mathrm{EME}$, in general, are not the two sides of the same coin; numerical and theoretical studies of fracture dynamics; the behavior of the elastic modulus of heterogeneous media during the fracture process; the discrimination of different thresholds of concentration of defects in a medium; critical phenomena; and micro-mechanics of granular materials - support the above conclusion. We underline that the EM silence before an EQ is not a puzzling but an expected feature.

In this contribution, we have based our analysis on the two-stage model for fracture-induced EME presented in Sect. 2, and we have presented much strong evidence that supports the specific hypothesis. However, it should be noted that other hypotheses for the origin of the observed pre-EQ EME have also been expressed that do not agree with the hypothesis of fracture-induced EME. For example, it has been proposed that pre-EQ EME originate from discharges in the atmosphere modified by the pre-EQ effects (e.g., Sorokin et al., 2011). Which hypothesis is true will be proved in the future, as the corresponding research evolves.

A crucial question arises immediately after the occurrence of a significant EQ referring to whether the EQ that occurred was the main shock or a foreshock of an ensuing larger EQ.

$\mathrm{AE}$ and EME studies of cracking in rocks have demonstrated that, in general, during cyclic loading, the level of $\mathrm{AE}$ and EME increases significantly when the stress exceeds the maximum previously reached stress level (Khatiashvili, 1984; Chelidze, 1986, and references therein; Li and Nordlund, 1993; Lavrov, 2005, and references therein; Mori and Obata, 2008; Mavromatou et al., 2008; Shkuratnik and Lavrov, 2002). This phenomenon was first reported in metals (Kaiser, 1953) and is now known as the Kaiser "stressmemory" effect. If we accept that the EME Kaiser effect is extended to the geological scale, then we suggest the following hypothesis should be thoroughly investigated in the future:

An observed continuous absence of fracture-induced $\mathrm{MHz}-\mathrm{kHz}$ EME after the occurrence of a significant EQ implies that the launched significant EQ was the main shock. If a new MHz-kHz EME sequence emerges during a time period of a few tens of hours after the already occurred significant EQ, this means that a new significant EQ is preparing, however in a new undamaged region of the Earth's crust out of the fractured region of the already occurred EQ.

Acknowledgements. Research co-funded by the EU (European Social Fund) and national funds, action "Archimedes III-Funding of research groups in T.E.I.", under the Operational Programme "Education and Lifelong Learning 2007-2013".

Edited by: D. Keefer

Reviewed by: K. Baddari and three anonymous referees

\section{References}

Allegre, C., Le Mouell, J., and Provost, A.: Scaling rules in rock fracture and possible implications for earthquake prediction, $\mathrm{Na}$ ture, 297, 47-49, 1982.

Alonso-Marroquin, F., Vardoulakis, I., Herrmann, H., Weatherley, D., and Mora, P.: Effect of rolling on dissipation in fault gouges, Phys. Rev. E, 74, 031306, 10 pp., 2006.

Amitrano, D. and Helmstetter, A.: Brittle creep, damage, and time to failure in rocks, J. Geophys. Res., 111, B11201, doi:10.1029/2005JB004252, 2006.

Anoosherhpoor, A. and Brune, J.: Frictional heat generation and seismic radiation in a foam rubber model of earthquakes, Pure Appl. Geophys., 142, 735-747, 1994.

Arbadi, S. and Sahimi, M.: Test of universality for threedimensional models of mechanical breakdown in disordered solids, Phys. Rev. B, 41, 772-775, 1990.

Astrom, J., Herrmann, H., and Timonen, J.: Granular packing and fault zone, Phys. Rev. Lett., 84, 638-641, 2000. 
Baddari, K. and Frolov, A.: Regularities in discrete hierarchy seismo-acoustic mode in a geophysical field, Ann. Geophys.Italy, 53, 31-42, 2010.

Baddari, K., Sobolev, G. A., Frolov, A. D., and Ponomarev, A. V.: An integrated study of physical precursors of failure in relation to earthquake prediction, using large scale rock blocks, Ann. Geophys.-Italy, 42, 771-787, doi:10.4401/ag-3758, 1999.

Baddari, K., Frolov, A., Tourtchine, V., and Rahmoune, F.: An integrated study of the dynamics of electromagnetic and acoustic regimes during failure of complex macrosystems using rock blocks, Rock Mech. Rock Eng., 44, 269-280, 2011.

Bahat, D., Frid, V., Rabinovitch, A., and Palchik, V.: Exploration via electromagnetic radiation and fractographic methods of fracture properties induced by compression in glass-ceramic, Int. J. Fracture, 116, 179-194, 2002.

Mahmoodi Baram, R., Herrmann, H. J., and Rivier, N.: Spacefilling bear- ing in three dimensions, Phys. Rev. Lett., 92, 044301, doi:10.1103/PhysRevLett.92.044301, 2004.

Benioff, H.: Earthquakes and rock creep, Part I: Creep characteristics of rocks and the origin of aftershocks, B. Seismol. Soc. Am., 41, 31-62, 1951.

Bowman, D., Ouillon, G., Sammis. C., Sornette, A., and Sornette, D.: An observational test of the critical earthquake concept, J. Geophys. Res., 103, 24359-24372, doi:10.1029/98JB00792, 1998.

Buchel, A. and Sethna, J. P.: Statistical mechanics of cracks: Fluctuations, breakdown, and asymptotics of elastic theory, Phys. Rev. E, 55, 7669-7690, 1997.

Carpinteri, A.: Mechanical Damage and Crack Growth in Concrete: Plastic Collapse to Brittle Fracture, Martinus Nijhoff Publishers, Dordrecht, 1986.

Carpinteri, A.: Cusp catastrophe interpretation of fracture instability, J. Mech. Phys. Solids, 37, 567-582, 1989.

Carpinteri, A.: A catastrophe theory approach to fracture mechanics, Int. J. Fracture, 44, 57-69, 1990.

Carpinteri, A.: Scaling laws and renormalization groups for strength and toughness of disordered Materials, Int. J. Solids Struct., 31, 291-302, 1994.

Carpinteri, A., Cornetti, P., and Sapora, A.: Brittle failures at rounded V-notches: a finite fracture mechanics approach, Int. J. Fracture, 172, 1-8, 2011.

Carpinteri, A., Lacidogna, G., Manuello, A., Niccolini, G., Schiavi, A., and Agosto, A.: Mechanical and electromagnetic emissions related to stress-induced cracks, Exp. Techniques, 36, 5364, doi:10.1111/j.1747-1567.2011.00709.x, 2012.

Chakrabarti, B. and Benguigui, L., Statistical Physics of Fracture and Breakdown in Disordered Systems, Oxford University Press, Oxford, 1998.

Chauhan, V. and Ashok Misra: Effects of strain rate and elevated temperature of electromagnetic radiation emission during plastic deformation and crack propagation in ASTM B 265 grade 2 Titanium sheets, J. Mater. Sci., 43, 5634-5643, doi:10.1007/s10853008-2590-5, 2008.

Chen, Y. Z.: A novel solution for effective elastic moduli of 2D cracked medium, Eng. Fract. Mech., 84, 123-131, 2012.

Chelidze, T. L.: Percolation model of fracture of solids and earthquake prediction, Reports of Acad. Sci. USSR, 246, 51-54, 1979 (in Russian).
Chelidze, T.: Theory of percolation and fracture of rocks, in: Proc. of VI All Union Conf. on Mech. of Rocks, Frunze, 1978, 107117, 1980b (in Russian).

Chelidze, T.: Percolation Theory in Mechanics of Geomaterials, Nauka, Moscow, 273 pp., 1987 (in Russian).

Chelidze, T.: Fractal damage mechanics of geomaterials, Terra Nova, 5, 421-437, doi:10.1111/j.1365-3121.1993.tb00280.x, 1993.

Chelidze, T., Reusche, T., Darot, M., and Gueguen, Y.: On the elastic properties of depleted refilled solids near percolation, J. Phys. C Solid State, 21, L1007, doi:10.1088/00223719/21/30/001, 1988.

Chelidze, T., Kolesnikov, Yu., and Matcharaahvili, T.: Seismological criticality concept and percolation model of fracture, Geophys. J. Int., 164, 125-136, 2006.

Chelidze, T. L.: The model of fracture process of solids, Solid State Phys., Moscow, 22, 2865-2866, 1980a.

Chelidze, T. L.: Percolation and fracture, Phys. Earth Planet. In., 28, 93-101, doi:10.1016/0031-9201(82)90075-9, 1982.

Chelidze, T. L.: Percolation theory as a tool for imitation of fracture process in rocks, Pure Appl. Geophys., 124, 731-748, doi:10.1007/BF00879607, 1986.

Chelidze, T. L. and Kolesnikov, Yu. M.: On the physical interpretation of a transitional amplitude in percolation theory, J. Phys. A., 17, L791, doi:10.1088/0305-4470/17/14/012, 1984.

Chelidze, T. L, Spetzler, H., Getting, I. C., and Avaliani, Z. A.: Experimental investigation of the elastic modulus of a fractal system - A model of fractured rock, Pure Appl. Geophys., 134, 31-43, doi:10.1007/BF00878078, 1990.

Cicerone, R. D., Ebel, J. E., and Britton, J.: A systematic compilation of earthquake precursors, Tectonophysics, 476, 371-396, 2009.

Contoyiannis, Y. F. and Eftaxias, K.: Tsallis and Levy statistics in the preparation of an earthquake, Nonlin. Processes Geophys., 15, 379-388, doi:10.5194/npg-15-379-2008, 2008.

Contoyiannis, Y., Kapiris, P., and Eftaxias, K.: A monitoring of a pre-seismic phase from its electromagnetic precursors, Phys. Rev. E, 71, 061123, 14 pp., 2005.

Cress, G., Brady, B., and Rowell, G.: Sources of electromagnetic radiation from fracture of rock samples in the laboratory, Geophys. Res. Lett., 14, 331-334, 1987.

Davidsen, J. and Schuster, H. G.: Simple model for $1 / f^{\alpha}$ noise, Phys. Rev. E, 65, 026120, 4 pp., doi:10.1103/PhysRevE.65.026120, 2002.

Dickinson, J. T., Langford, S. C., Jensen, L. C., McVay, G. L., Kelso, J. F., and Pantano, C. G.: Fractoemission from fused silica sodium silicate glasses, J. Vac. Sci. Technol. A, 6, 1084-1089, doi:10.1116/1.575646, 1998.

Diodati, P., Marchesony, F., and Piazza, S.: Acoustic emission from volcanic rocks: an example of self-organized criticality, Phys. Rev. Lett., 67, 2239-2249, 1991.

Eftaxias, K.: Are there pre-Seismic electromagnetic precursors? A multidisciplinary approach, in: Earthquake Research and Analysis - Statistical Studies, Observations and Planning, InTech, Chapter 11, 217-246, doi:10.5772/28069, 2012.

Eftaxias, K., Chelidze, T., and Surkov, V.: Thresholds for transport and fracture: implications for earthquakes precursors, EGU Geophysical Research Abstracts, 7, 01712, SRef-ID: 16077962/gra/EGU05-A-01712, 2005. 
Eftaxias, K., Kapiris, P., Dologlou, E., Kopanas, J., Bogris, N., Antonopoulos, G., Peratzakis, A., and Hadjicontis, V.: EM anomalies before the Kozani earthquake: A study of their behavior through laboratory experiments, Geophys. Res. Lett., 29, 69-1-69-4, doi:10.1029/2001GL013786, 2002.

Eftaxias, K., Kapiris, P., Polygiannakis, J., Peratzakis, A., Kopanas, J., Antonopoulos, G., and Rigas, D.: Experience of short term earthquake precursors with VLF-VHF electromagnetic emissions, Nat. Hazards Earth Syst. Sci., 3, 217-228, doi:10.5194/nhess-3-217-2003, 2003.

Eftaxias, K., Sgrigna, V., and Chelidze, T. (Eds): Mechanical and electromagnetic phenomena accompanying preseismic deformation: From laboratory to geophysical scale, Tectonophysics, 431, 1-5, doi:10.1016/j.tecto.2006.10.001, 2007.

Eftaxias, K., Athanasopoulou, L., Balasis, G., Kalimeri, M., Nikolopoulos, S., Contoyiannis, Y., Kopanas, J., Antonopoulos, G., and Nomicos, C.: Unfolding the procedure of characterizing recorded ultra low frequency, $\mathrm{kHZ}$ and $\mathrm{MHz}$ electromagetic anomalies prior to the L'Aquila earthquake as preseismic ones - Part 1, Nat. Hazards Earth Syst. Sci., 9, 19531971, doi:10.5194/nhess-9-1953-2009, 2009.

Eftaxias, K., Balasis, G., Contoyiannis, Y., Papadimitriou, C., Kalimeri, M., Athanasopoulou, L., Nikolopoulos, S., Kopanas, J., Antonopoulos, G., and Nomicos, C.: Unfolding the procedure of characterizing recorded ultra low frequency, $\mathrm{kHZ}$ and $\mathrm{MHz}$ electromagnetic anomalies prior to the L'Aquila earthquake as pre-seismic ones - Part 2, Nat. Hazards Earth Syst. Sci., 10, 275294, doi:10.5194/nhess-10-275-2010, 2010.

Eftaxias, K., Maggipinto, T., Meister, C.-V., and Katz, O. (Eds): Progress in the research on earthquakes precursors, Special Issue, Nat. Hazards Earth, Syst. Sci., http://www. nat-hazards-earth-syst-sci.net/special_issue125.html, 2011.

Frid, V., Rabinovitch, A., and Bahat, D.: Electromagnetic radiation associated with induced triaxial fracture in granite, Phil. Mag. Lett., 79, 79-86, 1999.

Frid, V., Bahat, D., Goldbaum, J., and Rabinovitch, A.: Experimental and theoretical investigation of electromagnetic radiation induced by rock fracture, Israel J. Earth Sci., 49, 9-19, 2000.

Fukui, K., Ocubo, S., and Terashima, T.: Electromagnetic Radiation from rock during uniaxial compression testing: the effects of rock characteristics and test conditions, Rock Mech. Rock Eng., 38, 411-423, 2005.

Gabrielov, A., Newman, W. I., and Turcotte, D. L.: An exactly soluble hierarchical clustering model: inverse cascades, selfsimilarity, and scaling, Phys. Rev. E, 60, 5293-5300, 1999.

Geller, R., Jackson, D., Kagan, Y., and Mulargia, F.: Earthquakes cannot be predicted, Science, 275, 1616-1617, 1997.

Gluzman, S. and Sornette, D.: Self-consistent theory of rupture by progressive diffuse damage, Phys. Rev. E, 63, 066129, 11 pp., 2001.

Gokhberg, M., Morgounov, V., and Pokhotelov, Q.: Earthquake Prediction. Seismo-electromagnetic Phenomena, Gordon and Breach Science Publishers, Amsterdam, 1995.

Gol'd, R. M., Markov, G. P., and Mogila, P. G.: Pulsed electromagnetic radiation of minerals and rock subjected to mechanical loading, Izv.-Phys. Solid Earth, 7, 109-111, 1975.

Gonzalez, A. and Pantano, C.: Fractoemission during crack propagation in glass, Appl. Phys. Lett., 57, 246-248, 1990.
Guarino, A., Ciliberto, S., Garcimartin, A., Zei, M., and Scorretti, R.: Failure time and critical behaviour of fracture precursors in heterogeneous materials, Eur. Phys. J. B, 26, 141-151, 2002.

Guéguen, Y., Chelidze, T., and Le Ravalec, M.: Microstructures, percolation thresholds, and rock physical properties, Tectonophysics, 279, 23-35, 1997.

Hallbauer, D. K., Wagner, H., and Cook, N. G. W.: Some observations concerning the microscopic and mechanical behaviour of quartzite specimens in stiff, triaxial compression tests, Int. J. Rock Mech. Min., 10, 713-726, doi:10.1016/01489062(73)90015-6, 1973.

Hansen, A. and Schmittbuhl, J.: Origin of the universal roughness exponent of brittle fracture surfaces:stress-weighted percolation in the damage zone, Phys. Rev. Lett., 90, 45504-45507, 2003.

Hayakawa, M.: Atmospheric and Ionospheric Electromagnetic Phenomena Associated with Earthquakes, Terrapub, Tokyo, 1999.

Hayakawa, M. (Ed.): Electromagnetic Phenomena Associated with Earthquakes, Transworld Research Network, Trivandrum 695 023 Kerala, India, 2009.

Hayakawa, M. and Fujinawa, Y.: Electromagnetic Phenomena Related to Earthquake Prediction, Terrapub, Tokyo, 1994.

Hayakawa, M. and Hobara, Y.: Current status of seismoelectromagnetics for short-term earthquake prediction, Geomatics, Natural Hazards and Risk, 1, 115-155, doi:10.1080/19475705.2010.486933, 2010.

Hayakawa, M. and Molchanov, O. (Eds): Seismo Electromagnetics, Lithosphere-Atmosphere-Ionosphere Couplimg, Terrapub, Tokyo, 2002.

Herrmann, H. and Roux, S.: Statistical Models for Fracture of Disordered Media, Elsevier, Amsterdam, 1990.

Herrmann, H., Astrom, J., and Baram, R.: Rotations in shear bands and polydisperse packing, Physica A, 344, 516-522, 2004.

Huang, J. and Turcotte, D.: Fractal distributions of stress and strength and variations of b-value, Earth Planet. Sc. Lett., 91, 223-230, 1988.

Hudson, J. A., Crouch, S. L., and Fairhurst, C.: Soft, stiff and servocontrolled testing machines: A review with reference to rock failure, Eng. Geol., 6, 155-189, 1972.

Kaiser, J.: Erkenntnisse und Folgerungen aus der Messung von Geräuschen bei Zugbeanspruchung von metallischen Werkstoffen, Verlag Stahleisen, Archiv für das Eisenhüttenwesen, 24, 4345, 1953 (in German).

Kapiris, P. G., Eftaxias, K. A., and Chelidze, T. L.: Electromagnetic signature of prefracture criticality in heterogeneous media, Phys. Rev. Lett., 92, 065702, doi:10.1103/PhysRevLett.92.065702, 2004.

Khatiashvili, N.: The electromagnetic effect accompanying the fracturing of alcaline-halide crystals and rocks, Izv.-Phys. Solid Earth, 20, 656-661, 1984.

Koktavy, P., Pavelka, J., and Sikula, J.: Characterization of acoustic and electromagnetic emission sources, Meas. Sci. Technol., 15, 973-977, 2004.

Kossobokov, V. G.: Testing earthquake prediction methods: "the West Pacific short-term forecast of earthquakes with magnitude MwHRV $\geq 5.8$ ", Tectonophysics, 413, 25-31, doi:10.1016/j.tecto.2005.10.006, 2006.

Kossobokov, V. G., Maeda, K., and Uyeda, S.: Precursory of seismicity in advance of the Kobe,1995, $\mathrm{M}=7.2$ Earthquake, Pure 
Appl. Geophys., 155, 409-423, doi:10.1007/s000240050272, 1999.

Kuksenko, V., Tomilin, N., Damaskinskaya, E., and Lockner, D.: A two stage model of fracture of rocks, Pure Appl. Geophys., 146, 253-263, 1996.

Kuksenko, V., Tomilin, N., and Chmel, A.: The role of driving rate in scaling characteristics of rock fracture, J. Stat. Mech.-Theory E., 2005, P06012, doi:10.1088/1742-5468/2005/06/P06012, 2005.

Kuksenko, V., Tomilin, N., and Chmel, A.: The rock fracture experiment with a drive control: A spatial aspect, Tectonophysics, 431, 123-129, 2007

Kuksenko, V. S., Makhmudov, Kh. F., Mansurov, V. A., Sultanov, U., and Rustamova, M. Z.: Changes in structure of natural heterogeneous materials under deformation, J. Min Sci., 45, 355-358, 2009.

Kumar, R. and Misra, A.: Some basic aspects of electromagnetic radiation emission during plastic deformation and crack propagation in $\mathrm{Cu}-\mathrm{Zn}$ alloys, Mat. Sci. Eng. A-Struct., 454-455, 203210, doi:10.1016/j.msea.2006.11.011, 2007.

Kun, F. and Herrmann, H. J.: Transition from damage to fragmentation in collision of solids, Phys. Rev. E, 59, 2623-2632, 1999.

Lacidogna, G., Manuello, A., Durin, G., Niccolini, G., Agosto, A., and Carpinteri, A.: Acoustic and magnetic emissions as precursor phenomena in failure processes, in: Proc. of SEM Annual Conference \& Exposition on Experimental and Applied Mechanics, Albuquerque, NM, 1-4 June 2009, Paper No. 540, 2009.

Lacidogna, G., Manuello, A., Carpinteri, A., Niccolini, G., Agosto, A., and Durin, G.: Acoustic and electromagnetic emissions in rocks under compression, Experimental Mechanics on Emerging Energy Systems and Materials, vol. 5, Conference Proceedings of the Society for Experimental Mechanics Series, 57-64, doi:10.1007/978-1-4419-9798-2_8, 2011.

Lacidogna, G., Carpinteri, A., Manuello, A., Durin, G., Schiavi, A., Niccolini, G., and Agosto, A.: Acoustic and electromagnetic emissions as precursors phenomena in failure processes, Strain, 47(Suppl. 2), 144-152, 2011.

Langford, S., Dickinson, J., and Jensen, L.: Simultaneous measurements of the electron and photon emission accompanying fracture of single-crystal MgO, J. Appl. Phys., 62, 1437-1449, 1987.

Latham, S., Abe, S., and Mora, P.: Parallel 3-D Simulation of a fault gouge using the lattice solid model, Pure Appl. Geophys., 163, 1949-1964, 2006.

Lavrov, A.: Fracture-induced physical phenomena and memory effects in rocks: A review, Strain, 41, 135-149, 2005.

Lei, X. L. , and Satoh, T.: Indicators of critical point behavior prior to rock failure inferred from pre-failure damage, Tectonophysics, 431, 97-111, 2007.

Li, C. and Nordlund, E.: Deformation of brittle rocks under compression with particular reference to microcracks, Mech. Mater., 15, 223-239, 1993.

Lin, Q. X., Tham, L. G., Yeung, M. R., and Lee, P. K. K.: Failure of granite under constant loading, Int. J. Rock Mech. Min., 41, 362-362, doi:10.1016/j.ijrmms.2003.12.068, 2004.

Lockner, D. A. and Madden, T. R.: A multiple-crack model of brittle fracture 1. Non-time-dependent simulations, J. Geophys. Res., 96, 613-642, 1991.
Lockner, D. A. and Okubo P. G.: Measurements of frictional heating in granite, J. Geophys. Res., 88, 4313-4320, doi:10.1029/JB088iB05p04313, 1983.

Lockner, D. A., Byerlee, J. D., Kuksenko, V., Ponomarev, A., and Sidorin, A.: Observations of quasi-static fault growth, in: Fault Mechanics and Transport Properties of Rocks, edited by: Evans, B. and Wong, T.-F., Academic Press, San Diego, CA, 3-31, 1992.

Mandelbrot, B. B.: The Fractal Geometry on Nature, Freeman, NY, 1982.

Mandelbrot, B. B., Passoja, D. E., and Paullay, A. J.: Fractal character of fracture surfaces of metals, Nature, 308, 721-722, 1984.

Marder, M. and Fineberg, J.: How things break, Phys. Today, 49, 24-29, 1996.

Matcharashvili, T., Chelidze, T., Zhukova, N., and Mepharidze, E.: Investigation of acoustic emission accompanying stick-slip movement of rock samples at different stiffnesses of spring-block system, Tribol. Int., 44, 811-819, 2011.

Mavromatou, C., Tombras, G. S., Ninos, D., and Hadjicontis, V.: Electromagnetic emission memory phenomena related to LiF ionic crystal deformation, J. Appl. Phys., 103, 083518, doi:10.1063/1.2906346, 2008.

McGarr, A. and Fletcher, J. B.: Maximum slip in earthquake fault zones, apparent stress, and stick-slip friction, B. Seismol. Soc. Am., 93, 2355-2362, 2003.

McGarr, A., Fletcher, J. B., Boettcher, M., Beeler, N., and Boatwright, J.: Laboratory-based maximum slip rates in earthquake rupture zones and radiated energy, B. Seismol. Soc. Am., 100, 3250-3260, doi:10.1785/0120100043, 2010.

Minadakis, G., Potirakis, S. M., Nomicos, C., and Eftaxias, K.: Linking electromagnetic precursors with earthquake dynamics: an approach based on nonextensive fragment and self-affine asperity models, Physica A, 391, 2232-2244, 2012a.

Minadakis, G., Potirakis, S. M., Stonham, J., Nomicos, C., and Eftaxias, K.: The role of propagating stress waves in geophysical scale: Evidence in terms of nonextensivity, Physica A, 391, 5648-5657, doi:10.1016/j.physa.2012.04.030, 2012b.

Miroshnichenko, M. and Kuksenko, V.: Study of electromagnetic pulses in initiation of cracks in solid dielectric, Sov. Phys.-Sol. State, 22, 895-896, 1980.

Miura, T. and Nakayama, K.: Spectral analysis of photons emitted during scratching of an insulator surface by a diamond in air, J. Appl. Phys., 88, 5444-5447, 2000.

Mizutani, H., Ishido, T., Yokokura, T., and Ohnishi, S.: Electrokinetic phenomena associated with earthquakes, Geophys. Res. Lett., 3, 365-368, 1976.

Mizuno, Y. and Mizuno, T.: Photon emission from ice during fracture, Jpn. J. Appl. Phys., 41, L209-L211, 2002.

Mjachkin, V. I., Kostrov, B. V., Sobolev, G. A., and Shamina, O. G.: Fundaments of the Physics of the Earthquake Epicenter and the Earthquake Precursors, Moscow, Nauka, 1975 (in Russian).

Mogi, K.: Study of the elastic shocks caused by the fracture of heterogeneous materials and its relations to earthquake phenomena, Bull. Earthq. Res. Inst. Univ. Tokyo, 40, 125-173, 1962a.

Mogi, K.: Magnitude-frequency relations for elastic shocks accompanying fractures of various materials and some related problems in earthquakes, Bull. Earthq. Res. Inst. Univ. Tokyo, 40, 831853, $1962 b$.

Mogi, K.: Source locations of elastic shocks in the fracturing process in rocks, Bull. Earthquake Res. Inst., 46, 1103-1125, 1968. 
Mogi, K.: Earthquake Prediction, Academic Press, Tokyo, 1985.

Molchanov, O. A. and Hayakawa, M.: Generation of ULF electromagnetic emissions by microfracturing, Geophys. Res. Lett., 22, 3091-3094, doi:10.1029/95GL00781, 1995.

Molchanov, O. A. and Hayakawa, M.: Seismo Electromagnetics and Related Phenomena: History and Latest Results, Terrapub, Tokyo, 2008.

Moreno, Y., Gómez, J. B., and Pacheco, A. F.: Fracture and secondorder phase transitions, Phys. Rev. Lett., 85, 2865-2868, 2000.

Morgounov, V.: Relaxation creep model of impending earthquake, Ann. Geophis., 44, 369-381, 2001.

Morgounov, V. and Shakhraman'yan, M.: Problems of operative earthquake prediction, Geophysics, 349A, 1053-1056, 1996.

Mori, Y. and Obata, Y.: Electromagnetic emission and AE Kaiser Effect for estimating rock in-situ stress, Report of the Research Institute of Industrial Technology, Nihon University, No. 93, 2008.

Mori, Y., Saruhashi, K., and Mogi, K.: Acoustic emission from rock specimen under cyclic loading, in: Progress on Acoustic Emission VII, JSNDI, Tokyo, 173-178, 1994.

Mori, Y., Obata, Y., Pavelka, J., Sikula, J., and Lolajicek, T.: AE Kaiser effect and electromagnetic emission in the deformation of rock sample, in: DGZ-Proceedings BB 90-CD, EWGAE, Lecture 14, 157-165, 2004a.

Mori, Y., Obata, Y., Pavelka, J., Sikula, J., and Lolajicek, T.: AE Kaiser effect and electromagnetic emission in the deformation of rock sample, Journal of Acoustic Emission, 22, 91-101, $2004 \mathrm{~b}$.

Mori, Y., Sedlak, P., and Sikula, J.: Estimation of rock in-situ stress by acoustic and electromagnetic emission, Adv. Mat. Res., 1314, 357-362, 2006.

Mori, Y., Obata, Y., and Sikula, J.: Acoustic and electromagnetic emission from crack created in rock sample under deformation, Journal of Acoustic Emission, 27, 157-166, 2009.

Mourot, G., Morel, S., Bouchaud, E., and Valentin, G.: Scaling properties of mortar fracture surfaces, Int. J. Fracture, 140, 3954, 2006.

Muto, J., Nagahama, H., Miura, T., and Arakawa, I.: Frictional discharge at fault asperities: origin of fractal seismoelectromagnetic radiation, Tectonophysics, 431, 113-122, 2007.

Nitsan, V.: Electromagnetic emission accompanying fracture of quartz-bearing rocks, Geophys. Res. Lett., 4, 333-336, 1977.

Ohnaka, M.: Acoustic emission during creep of brittle rock, Int. J. Mech. Min., 20, 121-134, 1983.

Ohnaka, M. and Mogi, K.: Frequency characteristics of acoustic emission in rocks under uniaxial compression and its relation to the fracturing process to failure, J. Geophys. Res., 87, 38733884, doi:10.1029/JB087iB05p03873, 1982.

Ohnaka, M. and Shen, I.: Scaling of the shears rupture process from nucleation to dynamic propagation: Implications of geomagnetic irregularity of rupturing surfaces, J. Geophys. Res., 104, 817844, 1999.

O'Keefe, S. and Thiel, D.: A mechanism for the production of electromagnetic radiation during fracture of brittle materials, Phys. Earth Planet. In., 89, 127-135, 1995.

Papadimitriou, C., Kalimeri, M., and Eftaxias, K.: Nonextensivity and universality in the earthquake preparation process, Phys. Rev. E, 77, 036101, doi:10.1103/PhysRevE.77.036101, 2008.
Park, J.-W. and Song, J.-J.: Numerical method for determination of contact areas of a rock joint under normal and shear loads, Int. J. Rock Mech. Min., 58, 8-22, 2013.

Paterson, M.: Experimental Rock Deformation, Springer, BerkinNew York, 1978.

Ponomarev, A., Zavyalov, A., Smirnov, V., and Lockner, D.: Physical modelling of the formation and evolution of seismically active fault zones, Tectonophysics, 277, 57-81, 1997.

Potirakis, S. M., Minadakis, G., Nomicos, C., and Eftaxias, K.: A multidisciplinary analysis for traces of the last state of earthquake generation in preseismic electromagnetic emissions, Nat. Hazards Earth Syst. Sci., 11, 2859-2879, doi:10.5194/nhess-112859-2011, 2011.

Potirakis, S. M., Minadakis, G., and Eftaxias, K.: Analysis of electromagnetic pre-seismic emissions using Fisher information and Tsallis entropy, Physica A, 391, 300-306, doi:10.1016/j.physa.2011.08.003, 2012a.

Potirakis, S. M., Minadakis, G., and Eftaxias, K.: Relation between seismicity and pre-earthquake electromagnetic emissions in terms of energy, information and entropy content, Nat. Hazards Earth Syst. Sci., 12, 1179-1183, doi:10.5194/nhess-121179-2012, 2012b.

Potirakis, S. M., Minadakis, G., and Eftaxias, K.: Sudden drop of fractal dimension of electromagnetic emissions recorded prior to significant earthquake, Nat. Hazards, 64, 641-650, doi:10.1007/s11069-012-0262-x, 2012c.

Potirakis, S. M., Karadimitrakis, A., and Eftaxias, K.: Natural time analysis of critical phenomena: the case of prefracture electromagnetic emissions, CHAOS, 23, 023117 , doi:10.1063/1.4807908, 2013.

Pulinets, S. and Boyarchuk, K.: Ionospheric Precursors of Earthquakes, Springer, Berlin, 2004.

Qian, S., Yian, J., Cao, H., Shi, S., Lu, Z., Li, J., and Ren, K.: Results of the observations on seismo-electromagnetic waves at two earthquake areas in China, in: Electromagnetic Phenomena related to Earthquake Prediction, edited by: Hayakawa, M. and Fujinawa, Y., Terrapub, Tokyo, 205-211, 1994.

Rabinovitch, A., Bahat, D., and Frid, V.: Comparison of electromagnetic radiation and acoustic emission in granite fracturing, Int. J. Fracture, 71, R33-R41, 1995.

Rabinovitch, A., Bahat, D., and Frid, V.: Similarity and dissimilarity of electromagnetic radiation from carbonate rocks under compression, drilling and blasting, Int. J. Rock Mech. Min., 39, 125-129, 2002.

Rundle, J. B., Turcotte, D. L., Shcherbakov, R., Klein, W., and Sammis, C.: Statistical physics approach to understanding the multiscale dynamics of earthquake fault systems, Rev. Geophys., 41, 1019-1049, doi:10.1029/2003RG000135, 2003.

Sadovski, M. (Ed.): Electromagnetic Precursors of Earthquakes, Nauka Moscow, 1982.

Sammis, C. G. and Sornette, D.: Positive feedback, memory, and the predictability of earthquakes, P. Natl. Acad. Sci. USA, 99, 2501-2508, doi:10.1073/pnas.012580999, 2002.

Schiavi, A., Niccolini, G., Terrizzo, P., Carpinteri, A., Lacidogna, G., and Manuello, A.: Acoustic emissions at high and low frequencies during compression tests in brittle materials, Strain, 47, 105-110, 2011. 
Scholz, C. H.: The frequency magnitude relation of microfracturing in rock and its relation to earthquakes, B. Seismol. Soc. Am., 58, 399-415, 1968.

Scholz, C. H.: The Mechanics of Earthquakes and Faulting, Cambridge University Press, Cambridge, 1990.

Scholz, C. H.: Earthquakes and friction laws, Nature, 391, 37-42, doi:10.1038/34097, 1998.

Scholz, C. H., Sykes, L., and Aggarwall, Y.: Earthquake prediction: A physical basis, Science, 181, 803-809, 1973.

Sharon, E. and Fineberg, J.: Confirming the continuum theory of dynamic brittle fracture for fast cracks, Nature, 397, 333-335, 1999.

Shen, L. and Li, J.: A numerical simulation for effective elastic moduli of plates with various distributions and sizes of cracks, Int. J. Solids Struct., 41, 7471-7492, 2004.

Shklovsky, B.: Electronic Properties of Doped Semicomtuctors, Berlin, Springer, 1984.

Shkuratnik, V. L. and Lavrov, A. V.: Kaiser effect in rocks undergone polyaxial loading, J. Min. Sci., 38, 48-56, 2002.

Sobolev, G. A.: Fundamental of Earthquake Prediction, ERC, Moscow, 1995.

Sobolev, G. A. and Ponomarev, A. V.: Earthquake Physics and Precursors, Nauka, Moscow, 270 pp., 2003.

Sornette, A. and Sornette, D.: Earthquake rupture as a critical point: Consequences for telluric precursors, Tectonophysics, 179, 327334, 1990.

Sornette, D.: Earthquakes: from chemical alteration to mechanical rupture, Phys. Rep., 313, 237-291, 1999.

Sornette, D.: Critical Phenomena in Natural Sciences, Springer, 2000.

Sornette, D. and Andersen, J. V.: Scaling with respect to disorder in time-to-failure, Eur. Phys. J. B, 1, 353-357, 1998.

Sornette, D. and Sammis, C.: Complex critical exponents from renormalization group theory of Earthquakes: Implications for Earthquake predictions, J. Phys. I, 5, 607-619, 1995.

Sorokin, V. M., Ruzhin, Yu. Ya., Yaschenko, A. K., and Hayakawa, M.: Generation of VHF radio emissions by electric discharges in the lower atmosphere over a seismic region, J. Atmos. Sol.-Terr. Phy., 73, 664-670, 2011.

Stanchits, S., Vinciguerra, S., and Dresen, G.: Ultrasonic velocities, acoustic emission characteristics and crack damage of basalt and granite, Pure Appl. Geophys., 163, 975-994, doi:10.1007/s00024-006-0059-5, 2006.

Stauffer, D.: Introduction to Percolation Theory, Taylor, London, 1985.
Surkov, V., Uyeda, S., Tanaka, H., and Hayakawa, M.: Fractal properties of medium and seismoelectric phenomena, J. Geodyn., 33, 477-487, 2002.

Turcotte, D.: Fractals and Chaos in Geology and Geophysics, Cambridge University Press, 1997.

Turcotte, D. L., Newman, W. I., and Shcherbakov, R.: Micro and macroscopic models of rock fracture, Geophys. J. Int., 152, 718728, 2003.

Uyeda, S., Nagao, T., and Kamogawa, M.: Short-term earthquake prediction: Current status of seismo-electromagnetics, Tectonophysics, 470, 205-213, 2009.

Varotsos, P.: The physics of Seismic Electric Signals, Terrapub, Tokyo, 2005.

Varotsos, P., Sarlis, N., and Skordas, E. S.: Natural Time Analysis: The New View of Time, Springer, Berlin, 2011.

Veje, C. T., Howell, D. W., and Behringer, R. P.: Kinematics of a two-dimensional granular Couette experiment at the transition to shearing, Phys. Rev. E, 59, 739-745, 1999.

Welker, P. and McNamara, A.: Precursors of failure and weakening in a biaxial test, Granul. Matter., 13, 93-105, 2011.

Wilson, B., Dewers, T., Reches, Z., and Brune, J.: Particle size and energetics of gouge from earthquake rupture zones, Nature, 434, 749-752, 2005.

Wolibrandt, J., Bruckner, U., and Linke, E.: Investigation of mechanically induced excited states on cleavage planes of ionic crystals. I. Method and apparatus for charge and electron emission measurements, Phys. Status Solidi A, 77, 545-556, doi:10.1002/pssa.2210770218, 1983.

Yamada, I., Masuda, K., and Mizutani, H.: Electromagnetic and acoustic emission associated with rock fracture, Phys. Earth Planet. In., 57, 157-168, 1989.

Zavyalov, A. D. and Sobolev, G. A.: Analogy in precursors of dynamic events at different scales, Tectonophysics, 152, 277-282, 1988.

Zhang, J., Soutis, C., and Fan, J.: Strain energy release rate associated with local delamination in cracked composite laminates, Composites, 25, 851-862, 1994a.

Zhang, P., Li, N., Li, X.-B., and Nordlund, E.: Compressive failure model for brittle rocks by shear faulting and its evolution of strength components, Int. J. Rock Mech. Min., 46, 830-841, 2009.

Zhang, S., Cox, S. F., and Paterson, M. S.: The influence of room temperature deformation on porosity and permeability in calcite aggregates, J. Geophys. Res., 99, 15761-15775, 1994 b. 